\title{
Manifestaciones Orales en el Síndrome de Deleción 22q11
}

\author{
Oral Manifestations in 22q11 Deletion Syndrome
}

\author{
Adrián Curto Aguilera
}

CURTO, A. A. Manifestaciones orales en el síndrome de deleción 22q11. Int. J. Odontostomat., 7(2):225-228, 2013.

RESUMEN: Las supresiones intersticiales del cromosoma 22q11 son las microdeleciones más frecuentes observadas en humanos, con una frecuencia de aproximadamente 1:4000-1:8000 nacidos. Incluye diversos síndromes como el síndrome de DiGeorge y Cayler. Los pacientes con deleción 22q11 presentan diversas combinaciones de las siguientes entidades clínicas: defectos cardíacos congénitos, facies característica, malformaciones del paladar, hipoparatiroidismo, hipocalcemia, inmunodeficiencia congénita debido a la hipoplasia tímica, retraso del crecimiento y problemas psiquiátricos. La etiología en la mayoría de los pacientes es una deleción recurrente 3-Mb en la región 22q11. Los objetivos del estudio fueron examinar y describir las manifestaciones orales en las personas con síndrome de deleción $22 q 11$ y analizar sus condiciones médicas y sus implicaciones en odontología. Se realizó una búsqueda sistemática en Octubre de 2012 en la base de datos PubMed, Scielo y Cochrane Library con las palabras clave: 22q11 deletion syndrome, oral health y dentist. Se observó una alta prevalencia de anomalías dentales. Las alteraciones del esmalte son las más frecuentes. Se presenta mayor número de hipomineralizaciones que de hipoplasias del esmalte. El hipoparatiroidismo y/o hipocalcemia no se consideran los factores etiológicos en las alteraciones del esmalte y no existe relación directa entre las condiciones médicas sistémicas y las alteraciones del esmalte. En el síndrome de deleción 22q11 la cavidad oral está afectada por alteraciones en el esmalte, en la morfología de los dientes, en el número de dientes y en la erupción dental. Es importante conocer que los pacientes con síndrome de deleción $22 q 11$ suelen padecer malformaciones cardíacas congénitas y problemas inmunológicos.

PALABRAS CLAVE: síndrome de deleción 22q11, salud oral, odontología.

\section{INTRODUCCIÓN}

La microdeleción $22 q 11$ es una deleción genética frecuente con variabilidad fenotípica amplia. Engloba una serie de síndromes, entre los que destaca el síndrome de DiGeorge y el síndrome de Cayler. Constituye la alteración cromosómica más frecuente en el ser humano, 1 de cada 4000 - 8000 recién nacidos la padecen, siendo el $93 \%$ de los casos mutaciones de novo.

La manifestación clínica más frecuente que se observa en estos pacientes son las alteraciones cardiacas (se presentan en el $70-90 \%$ de los sujetos) (Salazar et al., 2011). Por orden de frecuencia le siguen otras anomalías como las malformaciones velopalatinas, la presencia de hipocalcemia (Mussai et al., 2008; Angle, 2007; Pusceddu et al., 2002), la agenesia del timo (McClarren et al., 2006; Piliero et al., 2004) y las alteraciones inmunológicas (Gennery, 2012).
A nivel del sistema endocrino, además de observarse hipocalcemia también se presenta frecuentemente hipoparatiroidismo (Guerrero Fernández et al., 2011) e hipotiroidismo (Preece \& Smith, 2002).

Las anomalías neurológicas incluyen el retraso psicomotor y los problemas de aprendizaje (Duijff et al., 2012) y anomalías cerebrales estructurales (Shashi et al., 2012). Incluso se describe la presencia de alteraciones psiquiátricas (Armando et al., 2012) pudiendo incluso desarrollarse en la edad adulta esquizofrenia (van Beveren et al., 2012; Bassett et al., 2005).

En el aparato respiratorio, al igual que en el sistema cardiovascular, es frecuente la aparición de alteraciones estructurales pudiendo comprometer la permeabilidad de las vías aéreas y desarrollando a posteriori una insuficiencia respiratoria (Azpilicueta Idarreta et al., 2012). 
Otras anomalías menores que también pueden presentarse pero en pequeña proporción son alteraciones nefrourológicas, esqueléticas, digestivas y oculares (Campbell et al., 2010).

A nivel del complejo maxilofacial se observa una facies característica, pabellones auriculares pequeños y contorno superior recto, fisuras palpebrales antimongoloides, telecantus y, en ocasiones, labio leporino (Shouman et al., 2003).

\section{MATERIAL Y MÉTODO}

Se ha realizado una revisión bibliográfica de los artículos indexados en la base de datos PubMed, SciELO y Cochrane Library sobre aquellos estudios publicados hasta Octubre de 2012 y que tratan sobre las implicaciones del síndrome de deleción 22q11 en odontología. Se emplearon las palabras clave: 22q11 deletion syndrome, oral health y dentist.

\section{RESULTADOS}

Tras el análisis de la literatura científica destaca el escaso número de artículos e investigaciones realizadas que se centren en la evaluación y descripción de las anomalías y alteraciones que se presentan a nivel orofacial en estos pacientes.

En la Tabla I se enumeran las principales manifestaciones a nivel oral que se observan con mayor frecuencia en pacientes afectados por el síndrome de deleción 22q11.

Nordgarden et al. (2012), evaluó la salud oral de 50 pacientes con síndrome de deleción 22q11. Observaron en un $15 \%$ de sujetos la presencia de agenesias dentales. Un porcentaje elevado de dientes presentaban alteraciones a nivel del esmalte, en primer lugar hipomineralización del esmalte seguido de hipoplasias. Las hiopmineralizaciones eran mas frecuentes en dentición permanente que en dentición temporal. No se encontraron correlaciones con las condiciones médicas a excepción de los que presentan anomalías congénitas cardíacas que presentan menos alteraciones del esmalte (Nordgarden et al.).

Una encuesta realizada por Klingberg et al.
(2010), a padres de niños afectados por el síndrome de deleción 22q11 demuestra la preocupación de éstos por la salud bucodental de sus hijos afirmando también la dificultad adicional para el correcto mantenimiento de la misma en estos pacientes.

Klingberg et al. (2002), evaluaron el estado oral de 53 pacientes. Al igual que Nordgarden et al., observaron un alto porcentaje de sujetos con hipomineralización del esmalte seguido de hipoplasia del mismo; sin embargo, no describen mayor prevalencia de hipomineralización del esmalte en dentición permanente sobre la dentición temporal. Afirman que la presencia de hipoplasia del esmalte se asocia con condiciones médicas tales como el parto prematuro y la presencia de malformaciones congénitas cardiacas mientras que la hipomineralización del esmalte se asocia con condiciones médicas más difusas como pueden ser infecciones recurrentes. También describen mayor prevalencia de agenesias dentales, retraso en la erupción y alteraciones en el tamaño de los dientes (hipodoncia) con respecto a la población general. Se observa una mayor predisposición a padecer caries debido a las alteraciones en el esmalte que se presentan (Klingberg et al., 2002).

En el área orofacial se observa una prevalencia alta de labio leporino en estos pacientes al igual que ocurre con la presencia de paladar hendido.

Todos los niños con labio leporino y/o paladar hendido rutinariamente deben hacerse una prueba FISH 22q11 en vista de las consecuencias de un diagnóstico de síndrome velocardiofacial (Bashir et al., 2008; Harris, 2005; Brøndum-Nielsen et al., 1996). Sin embargo, la prevalencia de fisura palatina no es mayor en pacientes con este síndrome (Mingarelli et al., 1996).

Tabla I. Manifestaciones orales en el síndrome de deleción 22q11.

\begin{tabular}{l}
\hline Hipomineralización del esmalte \\
\hline Hipoplasia del esmalte \\
Agenesia dental \\
Retraso en la erupción dental \\
Alteraciones de la morfología dental: hipodoncia \\
Labio leporino \\
Paladar hendido \\
\hline
\end{tabular}




\section{CONCLUSIONES}

La microdeleción en el cromosoma 22q11 es un cuadro de anomalía del desarrollo caracterizado por una haploinsuficiencia de una región genómica en el cromosoma 22q11. Es la deleción intersticial más frecuente en los seres humanos.

No se observa relación entre las condiciones médicas presentes en estos pacientes y su estado de salud oral; sin embargo, es de destacar que los pacientes con anomalías congénitas cardíacas presentan menos alteraciones del esmalte.

Es importante conocer que estos pacientes presenta con alta frecuencia (entre el 70 y el $90 \%$ de los sujetos) alteraciones cardíacas al igual que alteraciones en el sistema inmunológico, resulta imprescindi- ble conocer este aspecto en relación a los tratamiento odontológicos que se practiquen.

En menor medida hay que considerar la presencia de hipocalcemia, hipoparatiroidismo e hipotiroidismo, entidades que tienen repercusiones en los tratamientos odontológicos que se realicen en estos pacientes.

Las alteraciones orofaciales que se observan con mayor frecuencia son la hipomineralización y la hipoplasia del esmalte, también es frecuente la presencia de agenesia dental, de alteraciones en la morfología dental (hipodoncia) y retraso en la erupción dental, se observa además con frecuencia labio leporino y paladar hendido.

CURTO, A. A. Oral manifestations in 22q11 deletion syndrome. Int. J. Odontostomat., 7(2):225-228, 2013.

ABSTRACT: Interstitial deletions of chromosome 22q11 are the most commonly seen microdeletions observed in humans, with a frequency of approximately 1:4000-1:8000 live births. It includes various syndromes such as DiGeorge and Cayler syndrome. Patients with the 22q11 deletion present various combinations of the following clinical features: congenital cardiac defects, characteristic facies, palate malformations, hypoparathyroidism, congenital hypocalcemia, immunodeficiency due to thymic hypoplasia, growth retardation and psychiatric problems. The etiology in the majority of patients is a 3-Mb recurrent deletion in region $22 q 11$. The aim of the study were to examine and describe oral manifestations in individuals with $22 q 11$ deletion syndrome and to analyze associations with medical conditions and the implications in dentist. A systematic search in October 2012 in PubMed, Scielo and Cochrane Library database with keywords: 22q11 deletion syndrome, oral health and dentist. Dental anomalies were registered in high numbers. Enamel disturbances were frequently seen. There were more hypomineralizations than hypoplasias. Hypoparathyroidism and/or hypocalcemia are not clear etiological factors for enamel disturbances and there were no major correlations between medical conditions and enamel disturbances. In $22 q 11$ deletion syndrome the oral cavity is affected by anomalies in dental enamel, tooth shape, numbers of teeth and eruption. It is important to know that patients with $22 q 11$ deletion syndrome often have congenital heart defects and immune problems.

\section{KEY WORDS: 22q11 deletion syndrome, oral health, dentist.}

\section{REFERENCIAS BIBLIOGRÁFICAS}

Angle, B. A 3-day-old infant with a congenital heart defect and hypocalcemia. 22q11 deletion syndrome. Pediatr. Ann., 36(5):275-6, 2007.

Armando, M.; Girardi, P.; Vicari, S.; Menghini, D.; Digilio, M. C.; Pontillo, M.; et al. Adolescents at ultra-high risk for psychosis with and without $22 \mathrm{q} 11$ deletion syndrome: $\mathrm{A}$ comparison of prodromal psychotic symptoms and general functioning. Schizophr. Res., 139(1-3):151-6, 2012.

Azpilicueta Idarreta, M.; Torres-Borrego, J.; Gilbert Pérez, J. J.; Ulloa Santamaría, E. \& Frías Pérez, M. 22Q11 microdeletion syndrome: cardio respiratory symptoms and fibrobronchoscopy. An. Pediatr. (Barc), 77(2):130-5, 2012.
Bashir, M. A.; Hodgkinson, P. D.; Montgomery, T. \& Splitt, M. 22q11 Deletion in children with cleft lip and palate--is routine screening justified? J. Plast. Reconstr. Aesthet. Surg., 61(2):130-2, 2008.

Bassett, A. S.; Chow, E. W.; Husted, J.; Weksberg, R.; Caluseriu, O.; Webb, G. D. \& Gatzoulis, M. A. Clinical features of 78 adults with 22q11 Deletion Syndrome. Am. J. Med. Genet. A., 138(4):307-13, 2005.

Brøndum-Nielsen, K. \& Christensen, K. Chromosome 22q11 deletion and other chromosome aberrations in cases with cleft palate, congenital heart defects and/or mental disability. A survey based on the Danish Facial Cleft Register. Clin. Genet., 50(3):116-20, 1996. 
Campbell, L.; McCabe, K.; Leadbeater, K.; Schall, U.; Loughland, C. \& Rich, D. Visual scanning of faces in 22q11.2 deletion syndrome: Attention to the mouth or the eyes? Psychiatry Res., 177(1-2):211-5, 2010.

Duijff, S. N.; Klaassen, P. W.; de Veye, H. F.; Beemer, F. A.; Sinnema, G. \& Vorstman, J. A. Cognitive development in children with 22q11.2 deletion syndrome. Br. J. Psychiatry, 200(6):462-8, 2012.

Gennery, A. R. Immunological aspects of 22q11.2 deletion syndrome. Cell. Mol. Life Sci., 69(1):17-27, 2012.

Guerrero Fernández, J.; Labrandero de Lera, C.; González Casado, I. \& Gracia Bouthelier, R. An adolescent with $22 q 11.2$ deletion syndrome and multiple endocrinopathies. An. Pediatr. (Barc), 74(5):327-31, 2011.

Harris, R. D. Obstetrics. 22q11 deletion syndrome with truncus arteriosus, hypoplastic left ventricle, VSD, clubfoot, sandal toes, cleft palate, butterfly vertebrae. Ultrasound Q., 21(2):107-8, 125-6, 2005.

Klingberg, G.; Hallberg, U. \& Oskarsdóttir, S. Oral health and $22 q 11$ deletion syndrome: thoughts and experiences from the parents' perspectives. Int. J. Paediatr. Dent., 20(4):283-92, 2010.

Klingberg, G.; Oskarsdóttir, S.; Johannesson, E. L. \& Norén, J. G. Oral manifestations in $22 q 11$ deletion syndrome. Int. J. Paediatr. Dent., 12(1):14-23, 2002.

McClarren, J.; Donnenfeld, A. E. \& Ravnan, J. B. Prenatal diagnosis of an unexpected interstitial 22q11.2 deletion causing truncus arteriosus and thymic hypoplasia in a ring 22 chromosome derived from a maternally inherited paracentric inversion. Prenat. Diagn., 26(13):1212-5, 2006.

Mingarelli, R.; Digilio, M. C.; Mari, A.; Amati, F.; Standoli, L.; Giannotti, A.; et al. The search for hemizygosity at 22qll in patients with isolated cleft palate. J. Craniofac. Genet. Dev. Biol., 16(2):118-21, 1996.

Mussai, F. J.; Cunningham, L. C.; Rezvani, G.; Stratakis, C. A.; Reynolds, J. C.; Nesterova, G.; et al. Hypocalcemia in a patient with osteosarcoma and 22q11.2 deletion syndrome. J. Pediatr. Hematol. Oncol., 30(8):612-7, 2008.

Nordgarden, H.; Lima, K.; Skogedal, N.; Følling, I.; Storhaug, K. \& Abrahamsen, T. G. Dental developmental disturbances in 50 individuals with the $22 q 11.2$ deletion syndrome; relation to medical conditions? Acta Odontol. Scand., 70(3):194-201, 2012.

Piliero, L. M.; Sanford, A. N.; McDonald-McGinn, D. M.; Zackai, E. H. \& Sullivan, K. E. T-cell homeostasis in humans with thymic hypoplasia due to chromosome 22q11.2 deletion syndrome. Blood, 103(3):1020-5, 2004.

Preece, J. M. \& Smith, R. A. Thyroid disease in children with 22q11.2 deletion syndrome. J. Pediatr., 141(2):297, 2002.

Pusceddu, M.; Bertone, A.; Campra, D.; Pontoriero, D. \& Guala, A. Transient neonatal hypocalcemia. Onset Manifestation of the 22q11.2 deletion síndrome. Minerva Pediatr., 54(4):343-5, 2002.

Salazar, M.; Villalba, G.; Mateus, H.; Villegas, V.; Fonseca, D.; Núñez, F.; et al. Analysis of microdeletions in 22q11 in Colombian patients with congenital heart disease. Invest. Clin., 52(4):334-43, 2011.

Shashi, V.; Veerapandiyan, A.; Keshavan, M. S.; Zapadka, M.; Schoch, K.; Kwapil, T. R.; et al. Altered Development of the Dorsolateral Prefrontal Cortex in Chromosome 22q11.2 Deletion Syndrome: An In Vivo Proton Spectroscopy Study. Biol. Psychiatry, 72(8):684-91, 2012.

Shouman, N.; Pabst, B.; Arslan-Kirchner, M.; Eckardt, A.; Schönweiler, R.; Ptok, M.; et al. Search for deletion $22 q 11.2$ in interphase nuclei of buccal mucosa of patients ascertained by isolated cleft palate: a new diagnostic approach. Int. J. Oral Maxillofac. Surg., 32(2):198-200, 2003.

van Beveren, N. J.; Krab, L. C.; Swagemakers, S.; Buitendijk, G. H.; Boot, E.; van der Spek, P.; et al. Functional geneexpression analysis shows involvement of schizophreniarelevant pathways in patients with 22 q11 deletion syndrome. PLoS One, 7(3):e33473, 2012.

Dirección de correspondencia:

Adrián Curto Aguilera

C/Eras, 4.

37710. Candelario

Salamanca

ESPAÑA

Tel.: 695914753

Email: adriancurtoaguilera@gmail.com

Recibido : 25-11-2012

Aceptado: 14-04-2013 